\title{
Development of Rest Facility Information Exchange System by Utilizing Delay Tolerant Network
}

\author{
Masahiro Ono \\ Department of Information and \\ Communication \\ Engineering, Tokyo Denki \\ University Tokyo, \\ Japan
}

\author{
Kei Sawai \\ Department of Information and \\ Communication \\ Engineering, Tokyo Denki \\ University Tokyo, \\ Japan
}

\author{
Tsuyoshi Suzuki \\ Department of Information and \\ Communication \\ Engineering, Tokyo Denki \\ University Tokyo, \\ Japan
}

\begin{abstract}
In this paper, we propose temporary rest facilities information exchange system among many people unable to get home by utilizing Delay Tolerant Network (DTN) after a disaster. When public transportation services are interrupted by the disaster, those people try to get home on foot while taking a rest at the facility. However, it is difficult for those people to obtain information of temporary rest facilities provided hurriedly, because communication infrastructures in the disaster area are disconnected by the disaster damage. Therefore, we propose a method to exchange the information among those people mutually by using mobile device via DTN for diffusion of the information. By using DTN, those people can communicate with each other by using mobile device and use the rest facility on the basis of the information even if the communication infrastructures are disconnected. Then, we develop mobile device application software to exchange the rest facility information among the people via DTN. In order to evaluate the application, we verified the communication performance in practical experiments. The experimental results showed the developed application had sufficient performance to exchange the information of the rest facility via DTN. Then, we verify the diffusivity of the rest facility information by a network simulation. The simulation results showed that the rest facility information was diffused widely and effectively to those people.
\end{abstract}

Keywords-Delay Tolerant Network; rest facility; disaster; communication infrastructure; simulation

\section{INTRODUCTION}

The problem of people (e.g. commuters, students, etc.) unable to get home after a disaster has attracted attention since the Great East Japan Earthquake. Public transportation services are suspended by disaster damages when a major disaster occurs. For example, in the Great East Japan Earthquake of 2011, a lot of railways in the metropolitan area suspended a passenger transport service for a long time [1]. In such case, it has been pointed out that a large number of people who have usually commuted by public transportation are unable to get home by public transportation unavailable.

Those people try to return to their home on foot, and spend a lot of time in walk. However, there are risks of secondary disasters including accidents caused by fatigue due to long-time walk; e.g. myocardial infarction, depression from mental stress and so on. Therefore, they have to need a rest on their way home. In fact, about 40,000 people in the metropolitan area took a rest on their way home after 5 hours in the Great East
Japan Earthquake [2]. Hence, as a facility to assist those people, rest facilities are prepared.

People unable to get home need information of those rest facilities; e.g. location, capacity, etc. Information of rest facilities is provided by prefectural and city governments in advance and it is shown on the disaster prevention map which was made in preparation for a disaster. However, in the large scale disaster, there are cases where new temporary rest facilities will be provided hurriedly since a rest facility is overcrowded more than a capacity of the facility [3] [4]. To spread information of these rest facilities not listed in the disaster prevention map to a large number of people, the use of mobile devices such as cell phones or smart phones that many people use are effective. On basis of this information, they are able to move to a temporary rest facility without being at a loss by using a map or GPS function provided in each device.

On the other hand, high degree of risk about disconnection of various communications infrastructure in urban area in a major disaster is known. In the Great East Japan Earthquake, about 1.94 million landlines function was suspended, and wave transmission was stopped in about 28,650 base station [5]. Furthermore, there are cases that the communication function is down by congestion even if the communication infrastructure is available. Therefore, under these situations, it is difficult to spread the information of temporary rest facilities to people unable to get home by the communication infrastructure. Hence, a diffusion method that can spread such information to those people even if the communication infrastructure is unavailable is needed.

As a communication method to transmission information under those situations, the Delay Tolerant Network (DTN) is useful [6]. DTN is a technique for performing communication in an environment in which End-to-End communication path is not always connected. DTN performs direct communication between devices by utilizing mobile devices. Figure 1 shows how to transfer the data by DTN. Store-and-forward scheme is mainly used for data transfer method in DTN. In store-andforward method, in order to send the data from a source to a destination, the mobile device stores the data if it cannot communicate with other devices, then the mobile device transfers the data if it can communicate with other devices. In this way, DTN can transmit information from a source to a destination by communicating with near devices while passing 
each other even if it is difficult to maintain communication connectivity continuously. DTN is suitable for rest facilities information transmission method in post-disaster situation because it can be used in portable smart phones.

In this research, we propose information exchange method to diffuse information of temporary rest facilities to people unable to get home by utilizing DTN in communication infrastructure unavailable after the large scale disaster.

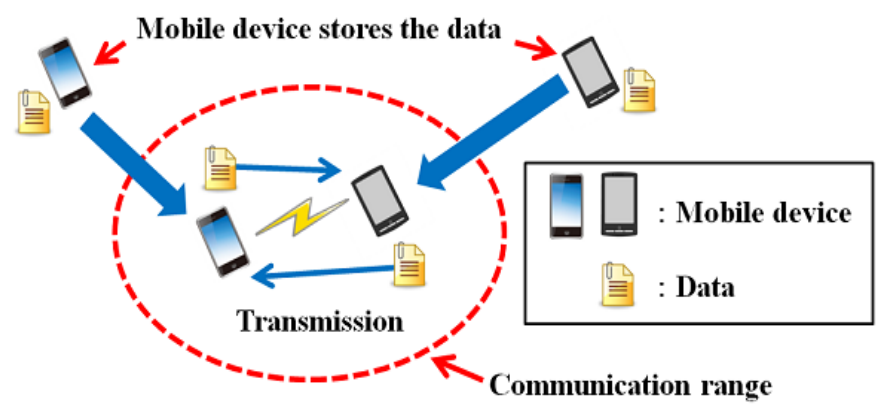

Fig. 1. Delay Tolerant Network

\section{RELATED WORKS}

A portable satellite communications system is developed by NTT Laboratories as an information transmission method of disaster [7]. This system provides the means of communication at rest facilities or shelters in a disaster. By using the vehiclemounted system with an antenna and telephone device in the post-disaster environment without the communication infrastructure, telephone call is possible via the satellite communications connection. However, a long distance communication is impossible when the base station is interrupted by the disaster damages because each base station has to be put within the communication range mutually. Further, it is difficult to transmit the rest facilities information for people unable to get home since a large number of people cannot use the system at a time. In addition, the movement of the vehicle-mounted system is difficult because it is considered that road of inner city is congested in a disaster.

As one of information transmission method of disaster, Heli-TV system is used by the disaster prevention center managed by Ministry of Land, Infrastructure, Transport and Tourism [8]. This system sends video data taken from a helicopter in the disaster area to disaster prevention center via satellite communications. A car equipped with satellite communication antenna moves in the post-disaster environment without communication infrastructure, and can relay the communication. However, it is difficult to utilize this system as a general communication system which sends the information to people unable to get home because this system is assumed to provide dedicated connection between the helicopter and the disaster prevention center. In addition, the movement of base station for this system by car is difficult because it is considered that road of inner city is congested in a disaster.

As a study utilizing DTN, MONAC of Android application was developed by Teranishi et al [9]. MONAC is a system that aims to transmit the disaster area information in an environment where communication infrastructure is disconnected by the disaster damage. MONAC exchanges the disaster area information between devices (e.g. mobile phone and smart phone) which have passed each other in the environment by DTN, then the information sends to Twitter when a device can connect to the communication infrastructure. However, MONAC assumes that the user is able to connect to the communication infrastructure in the mobile range; it might not work in the large environment where the communication infrastructure is interrupted.

A study of Data Gathering and Sharing based on DTN was proposed by Sun et al [10]. In this study, emergency personnel exchange the disaster area information with each other by utilizing DTN in the environment where the communication infrastructure is interrupted, and then the collected information is transmitted to the disaster response headquarters when the emergency personnel can connect the communication infrastructure. However, the proposed method assumes that the emergency personnel can connect to communication infrastructure in their moving range. Therefore, it might not work in the large environment where the communication infrastructure is interrupted.

In these studies, it is difficult to transmit information in the environment where the communication infrastructure is interrupted by disaster damages caused by the large scale earthquake. Therefore, we propose a system that can transmit the rest facility information to people unable to get home in such environment.

\section{PROPOSED SYSTEM}

\section{A. Assumed environment}

An assumed environment of the proposed system is large environment in which the communication infrastructure is disconnected by the disaster damage, and the communication function is stopped because of the communication amount increase. In such environment, information collection is difficult because communication services such as telephone, television broadcasting, e-mail and the Internet service cannot be used. In addition, many people in urban area cannot go back home for a long time because public transportation services are interrupted by disaster damages. Therefore, population density increases by the advent of people unable to get home on foot in urban area. These conditions show the increase of opportunity of communication by utilizing DTN since a large number of people pass each other on the sidewalk and those people move slowly by the traffic jam.

\section{B. Request of function}

This system aims to transfer the rest facility information to people unable to get home in the environment that has no communication infrastructure. Therefore, the communication method capable of transmitting information in such environment is needed. Further, this system is required to be used on the move because those people use it on their way home. It is also necessary to decide the rest facility information for the transmitting information source.

\section{Outline of system}

From the functional requirement in Section B, we propose the system to transmit the rest facility information which 
received in the rest facility to people unable to get home by utilizing DTN. DTN can transmit the rest facility information even in the environment that has no communication infrastructure because DTN is available on mobile devices and transmit the information by the device communication. In addition, DTN is useful as a method for long distance communication by exchanging the information among those people who go back home because it is possible to transmit the information to the distant with a device movement.

On basis of the survey report by Japanese cabinet office about necessary information for the people who went back home when the Great East Japan Earthquake had occurred, we decided the rest facility information to be transmitted is the information about position of the rest facility, capacity, presence or absence of relief supplies and toilet [11]. To find the rest facilities, the location information of the rest facilities is required. And, as information to determine the availability of rest facilities, it is necessary to transmit information about the capacity of the rest facility. In addition, in the case that the people have to walk for a long time to get home, information of the location of toilets is important. It is considered demand of toilets increases in the large scale disaster, because a lot of people walk for a long time to get home [12]. Therefore, the information about the number and the presence of toilets is important in supporting those people. There is a case that the relief supplies such as foods and beverages in the victim support facilities such as rest facilities are distributed. In a disaster, many stores are closed and vending machines don't work by the disaster damage. Further, it is difficult to ensure offer of foods and beverages due to the increase of the demand for foods and beverages by those people [13]. Therefore, the information about the presence of foods and beverages distribution is important in the use of rest facilities.

Figure 2 shows the overview of the system. If the communication infrastructure cannot connect by the disaster damage, the rest facility information transmission server is installed in the rest facility. If people unable to get home come to the rest facility, the server directly sends the rest facility information to their devices. The device of those people saves the received rest facility information. If those people exit the rest facility after taking a rest, their devices send the rest facility information to other persons' devices within communication range by utilizing DTN. The information is widely propagated by being transmitted to surrounding device in communication range from a device repeatedly via DTN.

Therefore, it is possible to transmit the rest facility information in the environment where the communication infrastructure is interrupted, and it is possible to search the rest facility by using the received rest facility information. The proposed system can transmit the rest facility information to people unable to get home, because a lot of people try to get home on foot in urban area in disaster situation. In this study, we develop functions to exchange the rest facility information by utilizing DTN between those people, and then we verify the diffusivity of the rest facility information by a simulation.

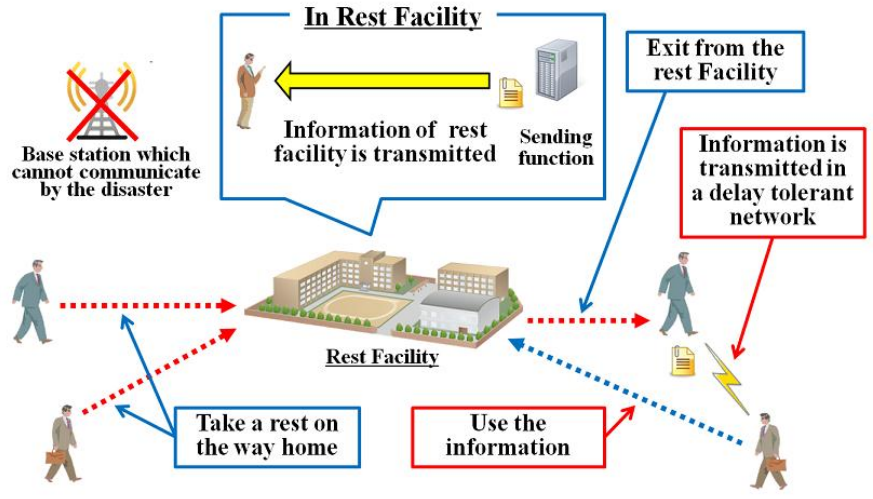

Fig. 2. System outline

\section{Development of DTN application}

In this system, the device needs to have functions of exchanging the rest facility information by utilizing DTN among people unable to get home. Therefore, the device to be used needs to have portability to be able to carry in a disaster, and the device-to-device communication function to communicate by utilizing DTN. Further, in this study, it is necessary to use the devices in which hardware and functions are relatively uniform because multiple devices are used. Therefore, in this study, we use the iPhone of Apple, Inc. in which these conditions are satisfied, and develop the system as the function of the $\mathrm{iOS}$ application.

Figure 3 shows the application of the algorithm. First, if the device detects the information transmission server in the rest facility by utilizing DTN, the device saves the rest facility information transmitted from the server. The device connects to the other devices when they were detected within communication range, and then the device transmits the rest facility information stored to other devices. The device, then, gets the rest facility information which had stored by other devices. If the device detects again other devices, it repeats the same operation from the detection of other devices of Figure 3.

Figure 4 shows the screen of the developed application. In the top part of the screen, there are the "add information screen" to enter the rest facility information and the "add button" to save the rest facility information. In the center part of the screen, there are the "start DTN" button to start the communication by utilizing DTN, the "finish DTN" button to terminate the DTN communication and the "log screen" that outputs an operation history of the application and communication status. In the lower part of the screen, there is the "information preservation screen" to output the rest facility information stored in the device. Application has the following three functions.

1) Registration of the rest facility information in the add information screen.

2) Transmission of the rest facility information stored to other devices by utilizing DTN.

3) Save of the rest facility information received from other devices. 
The application saves the rest facility information input in the add information screen to the database that was implemented in the application. And, the application transmits the rest facility information stored to other devices in the communication range by utilizing DTN. Then, the application stores the rest facility information received from other devices to the database. The stored rest facility information can be viewed in the information preservation screen. The transmission of the rest facility information by utilizing DTN and preservation of the rest facility information obtained is carried out automatically. The registration function of the rest facility information is necessary for the transmission information system. We equipped the application with the function (1) for the creation of the rest facility information. The function of (2) is capable of transmitting the rest facility information obtained from other devices by the function of (3). We employed the Bluetooth system as the communication system, because it is mounted on a lot of mobile devices and is battery friendly [14].

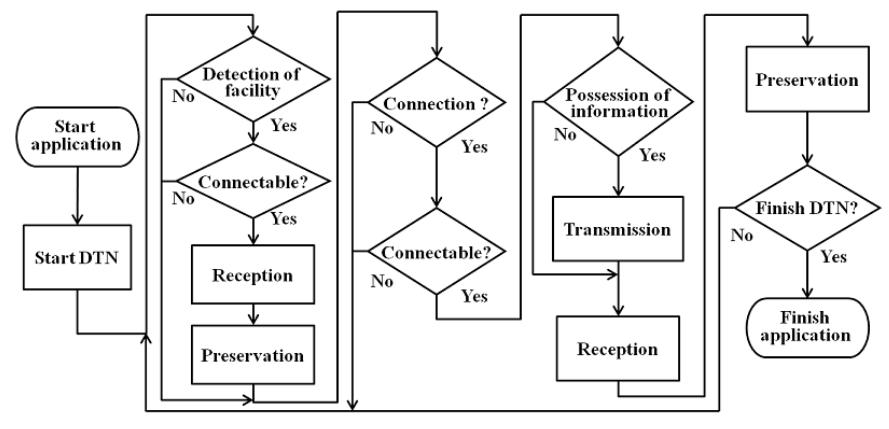

Fig. 3. Algorithm of DTN application

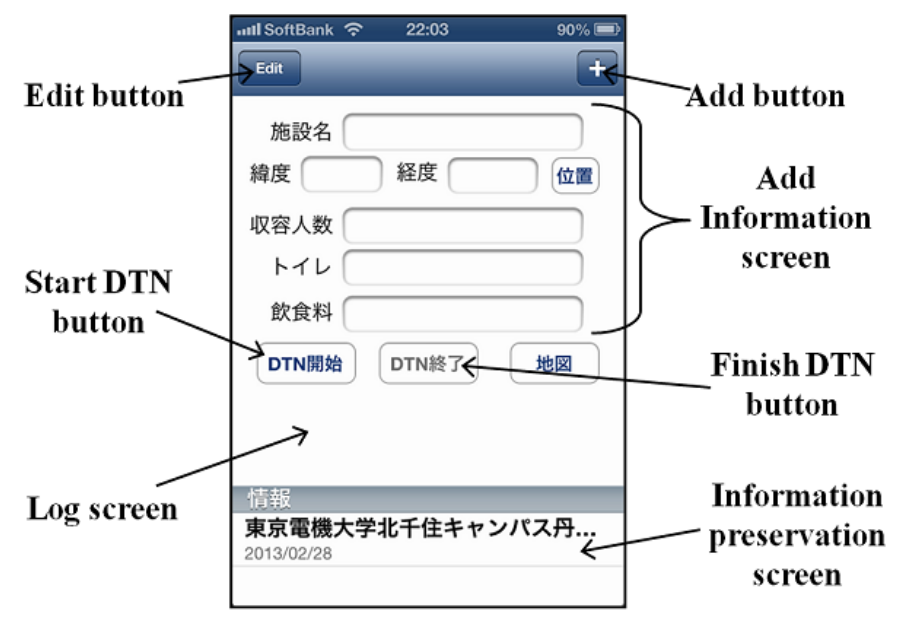

Fig. 4. iOS application

\section{Performance Evaluation Of Communication CONNECTIVITY OF DEVELOPED DTN APPLICATION}

\section{A. Experimental outline}

In this experiment, we verified the communication connectivity of our developed DTN application for sending and receiving the rest facility information. Figure 5 shows the experimental overview. The performance of the communication connectivity was evaluated by utilizing two
iPhone (Type: 3GS, 4S), it measured the processing time between two communication devices in the exchanging the rest facility information. Thus the processing time was measured by changing the distance between two communication devices. The processing time was defined the time between finish to receive the information and start to connect other device. We measured the processing time to the distance to disconnect area.

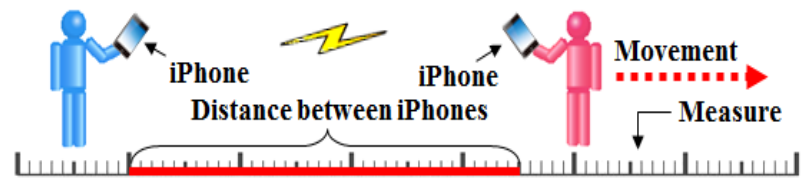

Fig. 5. Evaluation of communication quality

\section{B. Experimental conditions}

The experiment was executed at the corridor of kita-senju campus in Tokyo Denki University. There were not some obstacles in the movement line. The processing time was measured at $10[\mathrm{~m}]$ interval. The data size of sent information was configured to 1512 [Byte] by discussing the size of the rest facility information. The processing time was measured to 3 times in each distance, and then it was calculated the average value.

\section{Experimental results and Discussion}

Figure 6 shows the processing time of each measurement distance. The maximum distance of the DTN by utilizing our developed application was $90[\mathrm{~m}]$. In the average value of the processing time, iPhone $4 \mathrm{~S}$ spent to 5.65 [sec] and iPhone $3 \mathrm{GS}$ spent to 6.11 [sec].

The average of walking speed is defined to $4.0[\mathrm{~km} / \mathrm{h}]$ [15], and then the distance to walk in 6.11 [sec] is 6.79 [m]. Figure 7 shows the situation of the DTN in walking. Then the distance to walk in the processing time fit in the distance of the DTN. Therefore we confirmed that the DTN was able to exchange the rest facility information in walking situation.

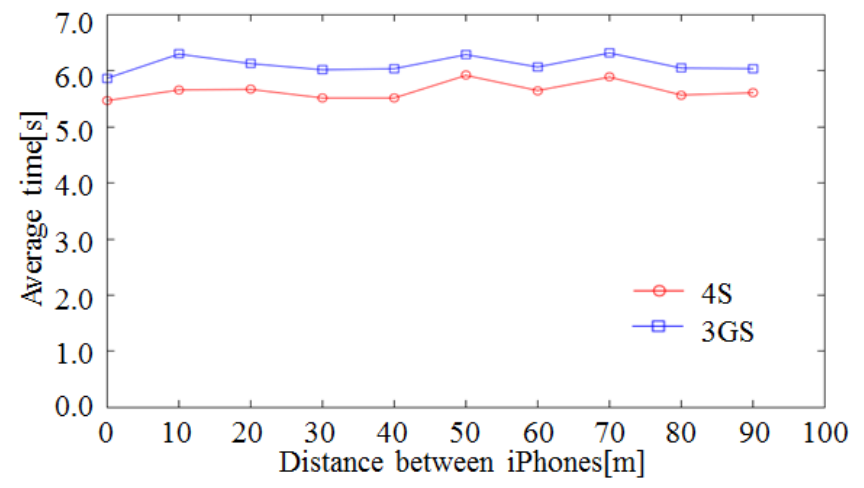

Fig. 6. Experimental result of communication quality

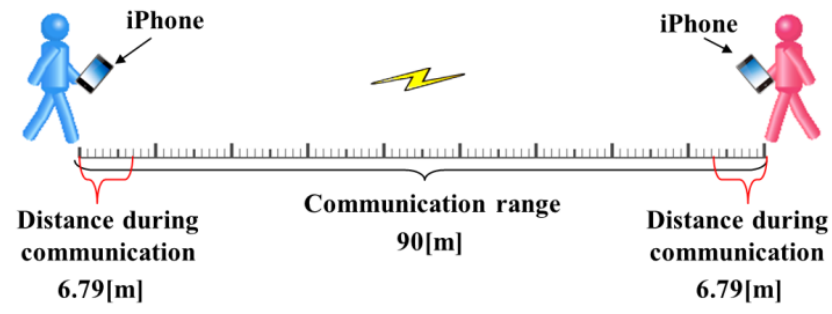

Fig. 7. Verification of communication quality 


\section{Verification Of Dtn Connection At The Time OF THE WALK}

\section{A. Experimental overview}

We verified exchange of the rest facility information between two pedestrians passing each other by utilizing our developed application in walking. Figure 8 shows the schematic diagram of the experiment. In this experiment, we used two iPhone (Type: 3GS, 4S) as communication device. Device holder started walking from out of communication range measured in previous chapter, and then we evaluated the capability of exchange of the rest facility information by utilizing DTN.

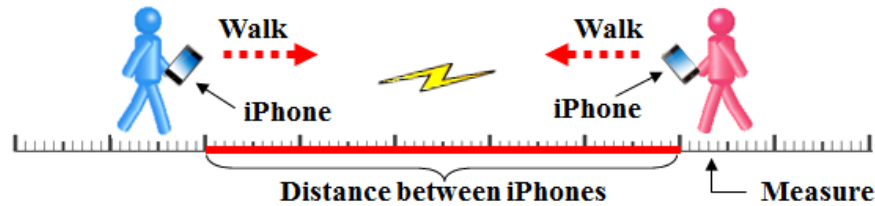

Fig. 8. Evaluation of proposed DTN application

\section{B. Experimental conditions}

The experiment was executed at the corridor of kita-senju campus in Tokyo Denki University. In this experiment, the device holders activates the function of DTN by pressing the start DTN button of application in the out of communication range, and then the terminal holders start walking. We experimented in the environment that does not have an obstacle on a straight line between the devices. We measured the distance between two devices from the start to the finish of communication to other device and the processing time. These parameters were measured to 5 times.

\section{Experimental results and Discussion}

The experimental results showed that developed application could exchange the information by utilizing DTN in the between two pedestrian. Figure 9 shows the measurement value of the processing time and the end-to-end distance from the start to the finish of communication. Exchanging information was finished before device holders passing each other. Processing time of this experiment shows a value close to the results of previous chapter. In addition, the detectable distance between two devices was shorter than the experimental results in previous chapter. We considered that the detection of the other device is difficult due to move two devices. However, the performance of DTN is sufficient values, because exchanging information was finished before device holders passing each other.

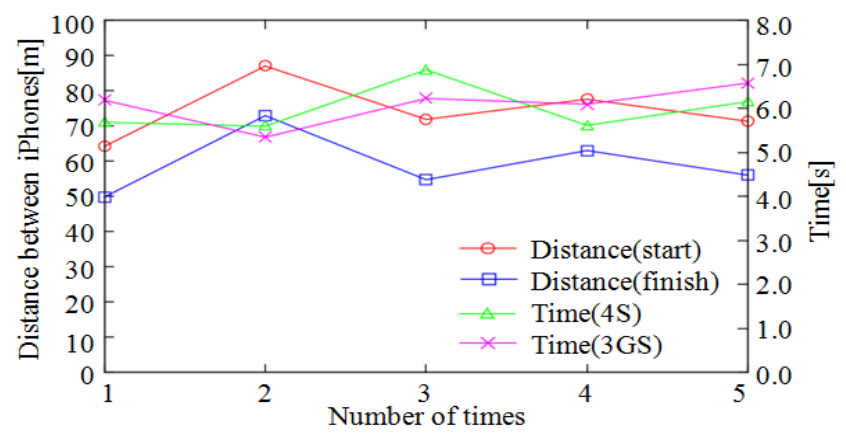

Fig. 9. Experimental result of DTN application

\section{Performance Evaluation By Using ENVIRONMENTAL SIMULATION}

\section{A. Experimental overview}

To verifying the diffusivity of the rest facility information by utilizing our proposed method, it requires to evaluate the conveyance of information by connection DTN in the environment that has no communication infrastructure. However the evaluation of the information diffusivity for many people in actual environment is distant experiment. Therefore, we evaluated the conveyance of information in simulation test. Then we adopt the "Opportunistic Network Environment Simulator (The One simulator)" as DTN evaluation software [16]. The One simulator is able to configure the parameter of the wireless communication protocols, the mode of locomotion and the number of people, etc., and then it simulate the migration of various node and the conveyance of information in the setup map. In this simulation test, we expanded capacity that setting the number of communication node in the One simulator.

Figure 10 shows the simulation overview by utilizing the One simulator. We simulated the information diffusivity from a rest facility by utilizing our proposed method in disaster area. People unable to get home is deployed on the setup map, and then randomly placed people moves on the setup pathway after running simulation. By the report of Japanese cabinet office, almost of those people started to move in after about 1 hour. Therefore we set those people with the rest facility information start to move in 1 hour after the start of simulation. The simulation time was set to 2 hours, we measured the number of those people conveyed the rest facility information and the maximum distance that information has been received at 10 minute intervals.

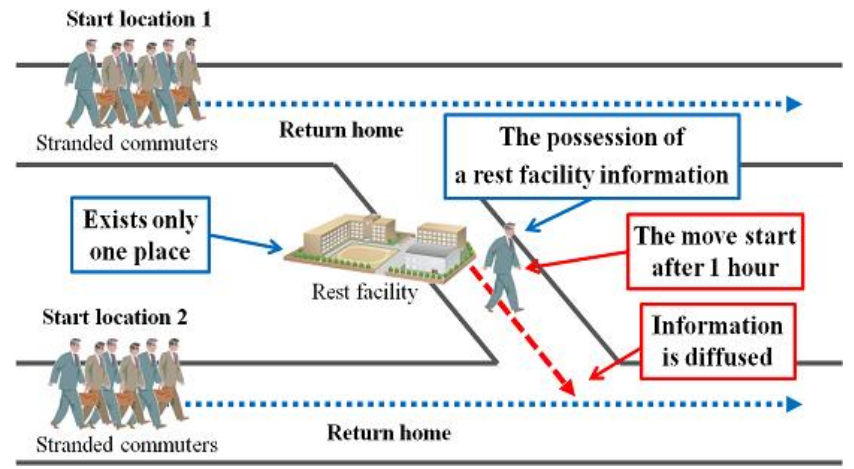

Fig. 10. Simulation of proposed system

\section{B. Experimental conditions}

The parameters configuration in simulation test was based on the data of people unable to get home about the Great East Japan Earthquake to reproduce the environment difficult get home. We are shown in the parameters details below. Simulation area was defined the Chiyoda city, Tokyo as the area of a lot of the population of daytime. The area with many people is considered to be vulnerable to damage [17].

Determination of the migration pass of those people is based on the "person trip survey" conducted by Tokyo metropolitan government [18]. "Person trip survey" is discussed the movement of people in Tokyo, it is configured 
the data that is the start and the finish point of traffic, the purpose of the movement, the means of transportation and the number of the people who are on the move. Then this survey involving the data that is the number of the person for the purpose of returning home is well suited to reproduce the activity of those people. In this study, we decided the start and the finish point of those people by the area data of "person trip survey", and then this data are reflected in simulation map.

The number of those people is also decided by "person trip survey". "Person trip survey" is the data at the time of no disaster. Therefore, we determined the number of people moving in per unit distance to get home by "person trip survey" and the data of the ratio of people that get home on foot in per unit distance in the Great East Japan Earthquake [19]. The number of the people who start returning home in per unit time of the simulation was defined by "person trip survey" and the data of the ratio of people that start returning home in per unit time in the Great East Japan Earthquake. Thus total number of people moving was defined as 7903 people in this simulation. Walking speed of the those people set to $4[\mathrm{~km} / \mathrm{h}]$ on the basis of the public data of Japanese cabinet office.

Then we set that every those people has an intelligent communication device, the entire device is installed our developed DTN application. The communication distance of the device set to 90 [m], communication time was configured to 5.65 [sec]. Communication protocol is adopted the Bluetooth, the number of device connecting at the same time set to 8 .

\section{Experimental results and Discussion}

Figure 11 shows the rate of the number that the person who received the rest facility information in the amount of people unable to get home. The number of the person who received the rest facility information based on the experimental result was 7871 people after 60 [min] from starting the information diffusion, and then the rate of received person was 99.6 [\%]. Moreover we confirmed that the diffused information was received to those people that is the rate of 86.9 [\%] in total number of people at 10 [min] after starting the information diffusion. The maximum propagation distance that was spread the information was shown to 2332.96 [m] in this simulation test. Then we confirmed that the our proposed DTN system was enable to spread the rest facility information to many those people, it was not needed enough time to spread the data in target area. This result was considered that the effect of staying a lot of those people in around environment of those people from starting simulation.

We considered the information diffusion capability of our proposed system in Tokyo metropolitan area by the experimental results. Area of the Tokyo metropolitan government without islands is $1782.89\left[\mathrm{~km}^{2}\right]$, and there is the rest facility of 1030 places [20] [21]. Therefore the area that a rest facility place superintends environment defined $1730961.17\left[\mathrm{~m}^{2}\right]$. Figure 12 shows the environment area. Diffusion of the rest facility information is significant to conveying the information to the distance more than the radius shown in Figure 12. In this simulation test, the maximum distance of the conveyed distance was measured to 2332.96 $[\mathrm{km}]$, thus the distance of the conveyed information was longer than the $742.47[\mathrm{~m}]$ as the radius.
Thus, it was considered that our proposed system diffused effectively to wide range by these results. Moreover the rate of the number of people unable to get home received the information was measured to 99.6 [\%] at 60 [min] after starting diffusion of the information. Therefore our proposed system was suggested to diffuse effectively to wide range without the environment existing communication infrastructure such as disaster area.

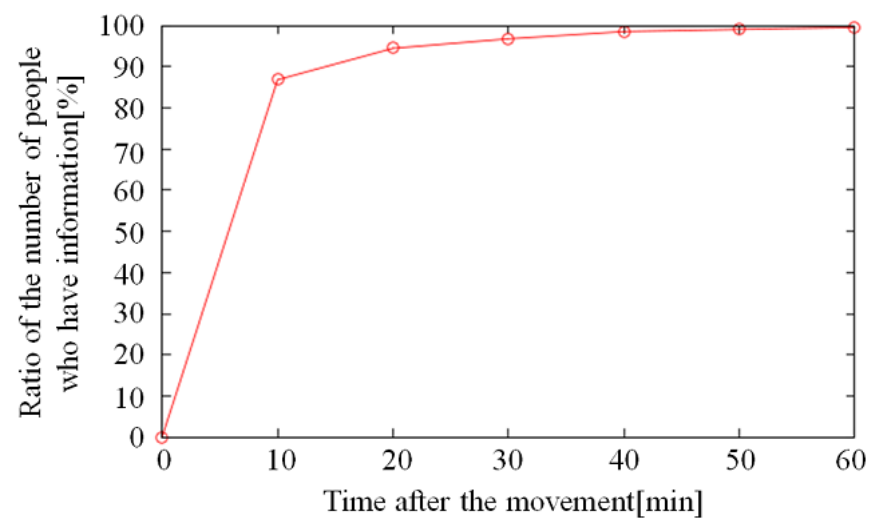

Fig. 11. Ratio of number of people who have information

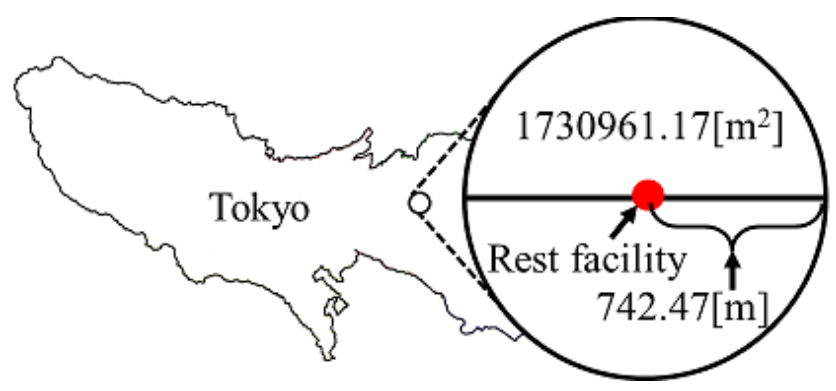

Fig. 12. Area to be responsible of rest facility

\section{CONCLUSION}

In this study, we proposed the exchange system of temporary rest facility information to people unable to get home by utilizing Delay Tolerant Network in urban area in post-disaster environment. The iOS application based on proposed method was developed, and then the communication performance was verified in the field test. In the test, we confirmed proposed method can exchange the temporary rest facility information between two pedestrian passing each other. Moreover, information diffusivity by the proposed method was verified by using the One Simulator. The result showed the proposed method is available for diffusion of the temporary rest facility information to a large number of people in urban area. In the simulation, we used data of Chiyoda-ku, a heavily populated city in Japan, for evaluation of proposed method by the One Simulator. Therefore, it is estimated that the information was frequently exchanged by many people passing each other.

In future work, to evaluate availability of the proposed method, the experiment should be carried out under several conditions; e.g. using data of sparsely populated area, changing walking speed of people on basis of a crowded situation, etc. Then we consider the simulation test estimate the precision 
result by referring the increase and decrease rate of density of population in chronological order.

\section{REFERENCES}

[1] Ministry of Land, Infrastructure, Transport and Tourism, http://www.mlit.go.jp/common/000208774.pdf, access: 10 January 2014.

[2] Mitsubishi Research Institute, http://www.mri.co.jp/NEWS/press/2011/2029010_1401.html, access: 10 January 2014.

[3] Setagaya

Ward

Office, http://www.city.setagaya.lg.jp/kurashi/104/141/563/571/573/d00033198 _d/fil/33198_1.pdf, access: 10 January 2014.

[4] Itabashi Ward Office, http://www.city.itabashi.tokyo.jp/c_kurashi/041/attached/attach_41697_ 1.pdf, access: 10 January 2014.

[5] Ministry of Internal Affairs and Communications, http://www.soumu.go.jp/main_content/000113017.pdf, access: 10 January 2014.

[6] K. Fall, "A delay-tolerant network architecture for challenged internets", Proceedings of the 2003 conference on Applications, technologies, architectures, and protocols for computer communications, pp.27-34, 2003.

[7] H. Takashi, O. Kazuhiko, I. Yutaka, Y. Hidekuni, "Satellite communications systems used in disaster recovery operations after the Great East Japan Earthquake and tsunami", The Institute of Electronics, Information andCommunication Engineers Technical Report, Satellite Telecommunications 111(336), pp.109-113, 2011.

[8] Ministry of Land, Infrastructure, Transport and Tourism, http://www.mlit.go.jp/bosai/disaster/bousaicenter/bousaicenter.htm, access: 10 January 2014.

[9] Y. Teranishi, S. Shimojo, "MONAC: SNS message dissemination over smartphone-based DTN and cloud", The IEEE International Conference on Peer-to-Peer Computing, pp158-159, 2011.
[10] W. Sun, T. Kitani, N. Shibata, K. Yasumoto, "A Data Gathering and Sharing Proposal for Disaster Relief based on DTN", The Special Interest Group Technical Reports of IPSJ, 2009(20), pp.61-66, 2009.

[11] Tokyo Metropolitan Gaverment, http://www.bousai.metro.tokyo.jp/japanese/kitaku_portal/tmg/pdf/9 11122 kaigi.pdf, access: 10 January 2014.

[12] Japan Toilet Labo, http://www.toilet.or.jp/dtinet/gaiyo.pdf, access: 10 January 2014.

[13] H. Nagato, N. Nishimura, K. Yamamoto, "Supporting Measures for the Difficult to Return Home at The Earthquake Disaster", Infrastructure Planning Review, No.22, pp.265-270, 2005.

[14] Agilent Technologies, http://cp.literature.agilent.com/litweb/pdf/59894204JAJP.pdf, access: 10 January 2014. Disaster Prevention Council, http://www.bousai.go.jp/kaigirep/chuobou/senmon/shutohinan/pdf/sanko 03.pdf, access: 10 January 2014.

[16] A. Keranen, J. Ott, and T. Karkkainen, "The ONE simulator for DTN protocol evaluation", International Conference on Simulation Tools and Techniques, No.55, pp.1-10, 2009.

[17] Tokyo Metropolitan Gaverment, http://www.toukei.metro.tokyo.jp/tyukanj/tj-index.htm, access: 10 January 2014.

[18] Tokyo Metropolitan Gaverment, http://www.tokyopt.jp/person/index.html, access: 10 January 2014.

[19] U. Hiroi, N. Sekiya, R. Nakajima, S.Waragai, H. Hanahara, "Questionnaire Survey concerning Stranded Commuters in Metropolitan Area in the East Japan Great Earthquake", Institute of Social Safety Science, No.15, pp.343-353, 2011.

[20] Tokyo Metropolitan Gaverment, http://www.metro.tokyo.jp/PROFILE/map_to.htm, access: 10 January 2014.

[21] Tokyo Metropolitan Gaverment, http://www.bousai.metro.tokyo.jp/japanese/tmg/pdf/240113kitakukihon. pdf, access: 10 January 2014 